\title{
New nanomedicines for treating atherosclerotic plaques
}

Atherosclerotic plaques are characterized by chronic inflammation that is often driven by macrophages and other myeloid cells. Two new nanoparticle-based therapies that target these inflammatory cells have now been independently developed that might combat early atherosclerotic disease or advanced atherosclerosis.

In the first study, researchers at Rutgers University, NJ, USA, developed sugarbased amphiphilic nanoparticles to specifically target scavenger receptors on macrophages that are recruited to sites of endothelial inflammation in response to deposition of oxidized LDL (oxLDL). Uptake of oxLDL molecules by scavenger receptors induces the conversion of macrophages to foamy cells, which drive an inflammatory cascade that leads to the development of atherosclerotic lesions.

The amphiphilic macromolecules were selected on the basis of their specificty of binding to scavenger receptors to competitively inhibit the binding and internalization of oxLDL. The amphiphilic molecules were then incorporated into serum-stable nanoparticles. In a mouse model of cardiovascular disease, whereby
ApoE-deficient mice were fed a Western diet for 8 weeks, four intravenous injections of the amphiphilic nanoparticles over a period of 25 days resulted in reduced lipid accumulation, neointimal hyperplasia and inflammatory signalling compared with treatment with control nanoparticles. Notably, the authors acknowledge that these particles are directed toward mechanisms that occur early in the development of atherosclerotic disease.

The second study, which was conducted by researchers at Columbia University, NY, USA, and Brigham and Women's Hospital, MA, USA, reported the development of collagen-IV-targeted nanoparticles containing a peptide (Ac2-26) that mimics the proresolving actions of annexin A1. In $\mathrm{Ldlr^{-/- }}$ mice fed a Western diet for 12 weeks, which is model for advanced atherosclerotic disease, Col-IV-Ac2-26 nanoparticles homed to atherosclerotic lesions, where Ac2-26, which was designed to be released over a 5 day period, suppressed generation of reactive oxygen species and increased production of IL-10. These atheroprotective effects were mediated in myeloid cells in an FPR2/ALX-dependent manner.

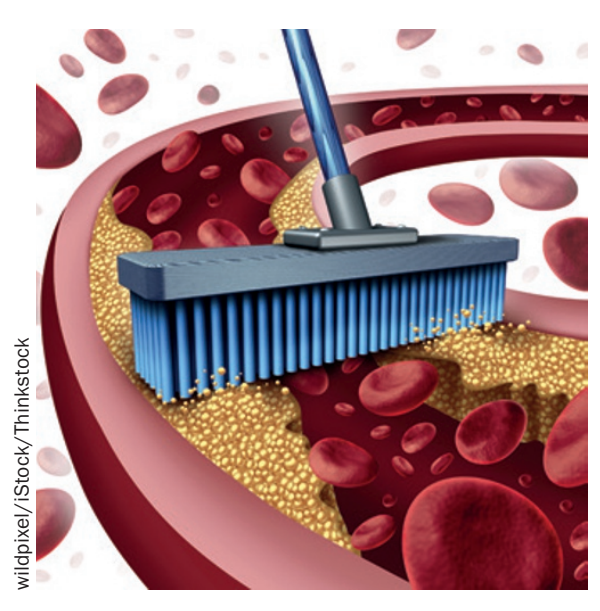

The authors speculate that these nanoparticles might represent a new modality with which to combat inflammation in atherosclerosis. Use of the nanoparticles might be associated with fewer adverse effects on the host defense system than directly targeting inflammatory mediators or their receptors.

Jennifer Sargent

Original articles Lewis, D. R. et al. Sugar-based amphiphilic nanoparticles arrest atherosclerosis in vivo. Proc. Natl Acad. Sci. USA doi:10.1073/pnas.1424594112 | Fredman, G. et al. Targeted nanoparticles containing the proresolving peptide Ac2-26 protect against advanced atherosclerosis in hypercholesterolemic mice. Sci. Transl. Med. 7 275ra20 (2015) 